GLASNIK MATEMATIČKI

Vol. 39(59)(2004), $55-72$

\title{
LOEWNER CHAINS AND PARAMETRIC REPRESENTATION OF BIHOLOMORPHIC MAPPINGS IN COMPLEX BANACH SPACES
}

\author{
Hidetaka Hamada* and Gabriela Kohr \\ Kyushu Kyoritsu University, Japan and Babeş-Bolyai University, Romania
}

Abstract. Let $X$ be a complex Banach space and let $B$ be the unit ball of $X$. In this paper we obtain sufficient conditions for biholomorphic mappings on $B$ to have parametric representation. Also we study certain properties of Loewner chains, and we obtain infinite dimensional versions of some well known univalence criteria on the unit ball of $\mathbb{C}^{n}$.

\section{INTRODUCTION AND PRELIMINARIES}

Let $X$ be a complex Banach space with respect to a norm $\|\cdot\|$. Let $B_{r}=\{z \in X:\|z\|<r\}$ and $B=B_{1}$. When $X=\mathbb{C}, B_{r}$ is denoted by $U_{r}$ and $B_{1}$ by $U$. If $G \subset X$ is a domain, let $H(G)$ be the set of holomorphic mappings from $G$ into $X$. If $f \in H(B)$, we say that $f$ is biholomorphic on $B$ if $f(B)$ is a domain and the inverse exists and is holomorphic on $f(B)$.

A mapping $v \in H(B)$ is called a Schwarz mapping if $v(0)=0$ and $\|v(z)\|<1, z \in B$.

Let $L(X, Y)$ denote the set of continuous linear operators from $X$ into another complex Banach space $Y$. Let $I$ be the identity in $L(X, X)$. For each $z \in X \backslash\{0\}$ let

$$
T(z)=\left\{l_{z} \in L(X, \mathbb{C}): l_{z}(z)=\|z\|,\left\|l_{z}\right\|=1\right\} .
$$

This set is nonempty by the Hahn-Banach theorem.

If $f \in H(B)$, we say that $f$ is normalized if $f(0)=0$ and $D f(0)=I$.

2000 Mathematics Subject Classification. 32H02, 30C45.

Key words and phrases. Biholomorphic mapping, Loewner chains, parametric representation, univalence criteria.

${ }^{*}$ Partially supported by Grant-in-Aid for Scientific Research (C) no. 14540195 from Japan Society for the Promotion of Science, 2003. 
Definition 1.1. Let $f: B \times[0, \infty) \rightarrow X$ be a mapping. We say that $f(z, t)$ is a Loewner chain if the following conditions hold:

(i) $f(\cdot, t)$ is univalent on $B, f(0, t)=0$ and $D f(0, t)=a(t) I, t \geq 0$, where $a(\cdot)$ is a continuous function on $[0, \infty)$ such that $a(t) \neq 0, t \geq 0,|a(\cdot)|$ $i s$ strictly increasing on $[0, \infty)$, and $a(t) \rightarrow \infty$ as $t \rightarrow \infty$;

(ii) there exists a univalent Schwarz mapping $v=v(z, s, t)$ (called the transition mapping associated to $f(z, t))$ such that $f(z, s)=f(v(z, s, t), t)$ for $z \in B$ and $0 \leq s \leq t<\infty$.

Note that the transition mapping $v=v(z, s, t)$ satisfies the condition $D v(0, s, t)=a(s) / a(t) I$ for $t \geq s \geq 0$, since $D f(0, t)=a(t) I$ and $a(t) \neq 0$ for $t \geq 0$.

If $f(z, t)$ is a Loewner chain such that $f(0, t)=0$ and $D f(0, t)=e^{t} I$ for $t \geq 0$, we say that $f(z, t)$ is a normalized Loewner chain.

A key role in our discussion is played by the sets

$$
\mathcal{N}=\left\{p \in H(B): p(0)=0, \operatorname{Re}\left[l_{z}(p(z))\right]>0, z \in B \backslash\{0\}, l_{z} \in T(z)\right\}
$$

and

$$
\mathcal{M}=\{p \in \mathcal{N}: D p(0)=I\} .
$$

Recently in [Ha-Ko2] we have proved the following useful result:

Lemma 1.2. Let $p \in \mathcal{M}$. Then for each $r \in(0,1)$ there exists $M=$ $M(r)>0$ (which is independent of $p$ ) such that $\|p(z)\| \leq M,\|z\| \leq r$.

This result leads to improvements in the existence theorem for the Loewner differential equation (see [Pf, Theorem 2.1], [Por2] and [Gr-Ha-Ko]).

The definition below generalizes the notion of close-to-starlikeness due to Pfaltzgraff and Suffridge [Pf-Su1] to infinite dimensions. To this end, we recall that if $f: B \rightarrow X$ is a holomorphic mapping such that $f(0)=0$, then $f$ is called starlike if $f$ is biholomorphic and $f(B)$ is a starlike domain with respect to zero.

Definition 1.3. Let $f: B \rightarrow X$ be a normalized holomorphic mapping. Also let $g: B \rightarrow X$ be a normalized starlike mapping. We say that $f$ is close-to-starlike with respect to $g$ if

$$
f(z, t)=f(z)+\left(e^{t}-1\right) g(z), \quad z \in B, t \geq 0,
$$

is a Loewner chain such that $f(\cdot, t)$ is biholomorphic on $B$ for each $t \geq 0$.

Hence, if $f$ is close-to-starlike, then $f$ is biholomorphic on $B$. Using an argument similar to the proof of [Pf-Su1, Theorem 2] (see also [Gr-Ha-Ko, Theorem 1.10]), based on the fact that $f(\cdot, t)$ is biholomorphic on $B$ for $t \geq 0$, we can show that if $f$ is close-to-starlike on $B$ with respect to a starlike mapping $g$, then there is a map $h=h(z, t)$ such that $h(\cdot, t) \in \mathcal{M}$ and

$$
e^{t} g(z)=D f(z, t) h(z, t), \quad z \in B, t \geq 0 \text {. }
$$


Letting $t=0$ in the above equation, we obtain

$$
\operatorname{Re}\left[l_{z}\left([D f(z)]^{-1} g(z)\right)\right]>0, \quad z \in B \backslash\{0\}, l_{z} \in T(z) .
$$

We remark that Pfaltzgraff and Suffridge [Pf-Su1] in their original paper used the above relation to define the notion of close-to-starlikeness in finite dimensional Banach spaces. They showed that the inequality (1.2) is equivalent to the fact that $f(z, t)$ given by $(1.1)$ is a Loewner chain. It would be interesting to see if the above relation is also sufficient to assure close-to-starlikeness in the case of infinite dimensional Banach spaces.

DEFINITION 1.4. Let $f: B \rightarrow X$ be a normalized locally biholomorphic mapping and let $\alpha \in(-\pi / 2, \pi / 2)$. We say that $f$ is spirallike of type $\alpha$ if $e^{-i \alpha}[D f(z)]^{-1} f(z) \in \mathcal{N}$.

We showed in the finite dimensional case that $f$ is spirallike of type $\alpha$ if and only if $f$ is biholomorphic on $B$ and each spiral $\exp \left(-e^{-i \alpha} t\right) f(z)(t \geq 0)$ is contained in $f(B)$ (see [Ha-Kol]).

In this paper we shall give an alternative characterization of spirallikeness of type $\alpha$ in terms of Loewner chains, and we shall obtain sufficient conditions for biholomorphic mappings on $B$ to have parametric representation. Also we study certain properties of Loewner chains, and we obtain infinite dimensional versions of some well-known univalence criteria on the unit ball of $\mathbb{C}^{n}$. To this end, we use the following results. For the proof of Lemma 1.5 it suffices to combine the proofs of [Por2, Lemma, Theorems 2, 3, 5 and Corollary 2], Lemma 1.2 and [Por3, Lemma 4.5] (cf. [Pf, Theorem 2.1]). Note that Poreda initially required the condition $\|h(z, t)\| \leq M(r)$ for $\|z\| \leq r$ and $t \geq 0$, where $M=M(r)$ is a positive constant. This condition is now clearly satisfied in view of Lemma 1.2.

LEMmA 1.5. Let $h: B \times[0, \infty) \rightarrow X$ satisfy the following assumptions:

(i) $h(\cdot, t) \in \mathcal{M}$ for $t \geq 0$;

(ii) $h$ is continuous on $B \times[0, \infty)$.

Then for each $s \geq 0$ and $z \in B$, the initial value problem

$$
\frac{\partial v}{\partial t}=-h(v, t), \quad t \geq s, v(s)=z,
$$

has a unique solution $v(t)=v(z, s, t)$ such that for fixed $s, t, v(\cdot, s, t)$ is a univalent Schwarz mapping such that $D v(0, s, t)=e^{s-t} I$. Also for each $z \in B$ and $s \geq 0$, there exists the limit

$$
\lim _{t \rightarrow \infty} e^{t} v(z, s, t)=f(z, s)
$$

which is a holomorphic and univalent mapping on $B$ such that $D f(0, s)=e^{s} I$ and $f(z, s)=f(v(z, s, t), t)$ for $z \in B$ and $0 \leq s \leq t<\infty$. Hence $f(z, t)$ is a 
normalized Loewner chain, which satisfies the growth result

$$
\frac{e^{t}\|z\|}{(1+\|z\|)^{2}} \leq\|f(z, t)\| \leq \frac{e^{t}\|z\|}{(1-\|z\|)^{2}}, z \in B, t \geq 0 .
$$

Moreover, if $f(z, \cdot)$ is differentiable on $[0, \infty)$ for each $z \in B$, then

$$
\frac{\partial f}{\partial t}(z, t)=D f(z, t) h(z, t), \quad z \in B, \quad t \geq 0 .
$$

The next result is due to Poreda [Por2, Theorem 6] and is a generalization to infinite dimensional Banach spaces of [Pf, Theorems 2.2 and 2.3] (in the case $X=\mathbb{C}$, compare with [Po, Theorem 6.2]).

LEMMA 1.6. Let $f: B \times[0, \infty) \rightarrow X$ be a continuous mapping such that $f(\cdot, t)$ is holomorphic on $B, f(0, t)=0, D f(0, t)=e^{t} I$ for $t \geq 0$, and $f(z, \cdot)$ is differentiable on $[0, \infty)$ for each $z \in B$. Also assume that there exist $r \in(0,1)$, $t_{0}>0$ and $M>0$ such that $\|f(z, t)\| \leq M e^{t}$ for $\|z\|<r$ and $t>t_{0}$. Further, suppose that there exists a mapping $h=h(z, t)$ which satisfies the assumptions (i) and (ii) of Lemma 1.5, such that

$$
\frac{\partial f}{\partial t}(z, t)=D f(z, t) h(z, t), \quad z \in B, \quad t \geq 0 .
$$

Then $f(z, t)$ is a Loewner chain and moreover, $f(z, s)=\lim _{t \rightarrow \infty} e^{t} v(z, s, t)$ for all $z \in B$ and $s \geq 0$, where $v=v(z, s, t)$ is the unique solution of the initial value problem (1.3).

We close this section with a result due to Poreda [Por2, Theorem 1], which yields that under certain assumptions Loewner chains satisfy the Loewner differential equation (for the finite dimensional case, see [Gr-Ha-Ko]).

LEMMA 1.7. Let $f=f(z, t)$ be a normalized Loewner chain and let $v=$ $v(z, s, t)$ be the transition mapping associated to $f(z, t)$. Assume there exist the derivatives $\partial f / \partial t$ for $t \geq 0$ and $\partial v / \partial t$ for $t=s \geq 0$, which are holomorphic on $B$. Then there exists a mapping $h=h(z, t)$ such that $h(\cdot, t) \in \mathcal{M}$ and

$$
\frac{\partial f}{\partial t}(z, t)=D f(z, t) h(z, t), \quad z \in B, \quad t \geq 0 .
$$

\section{MAin Results}

We begin this section with the following result, which is a generalization to infinite dimensions of [Pf, Theorem 2.1] (compare with [Por2, Lemma] and [Be, Lemma 1]).

TheOrem 2.1. Let $h: B \times[0, \infty) \rightarrow X$ satisfy the following assumptions:

(i) $h(\cdot, t) \in \mathcal{N}, D h(0, t)=c(t) I$, where $c:[0, \infty) \rightarrow \mathbb{C}$ is a continuous function such that

$$
\int_{0}^{\infty} \operatorname{Re} c(t) d t=\infty
$$


(ii) $h$ is continuous on $B \times[0, \infty)$.

Then for each $s \geq 0$ and $z \in B$, the initial value problem

$$
\frac{\partial v}{\partial t}=-h(v, t), \quad t \geq s, v(s)=z,
$$

has a unique solution $v(t)=v(z, s, t)$ such that for fixed $s, t, v(\cdot, s, t)$ is a univalent Schwarz mapping, $D v(0, s, t)=a(s) / a(t) I$, where $a(t)=$ $\exp \int_{0}^{t} c(\tau) d \tau$

ProOf. First we mention that $\operatorname{Re} c(t)>0$ for each $t \geq 0$ in view of $\left[\mathrm{Su}\right.$, Lemma 3] and the fact that $h(\cdot, t) \in \mathcal{N}$. Let $\alpha(t)=\int_{0}^{t} \operatorname{Re} c(\tau) d \tau$ and $\beta(t)=\int_{0}^{t} \operatorname{Im} c(\tau) d \tau$. Then

$$
a(t)=e^{\alpha(t)+i \beta(t)} \text { for } t \geq 0 .
$$

Clearly $a(\cdot) \in C^{1}([0, \infty)), a(t) \neq 0, t \geq 0,|a(t)| \rightarrow \infty$ as $t \rightarrow \infty$, and $|a(\cdot)|$ is strictly increasing on $[0, \infty)$. Also let

$$
z^{*}=e^{i \beta(t)} z \text { and } t^{*}=\alpha(t) \text {, for } z \in B \text { and } t \geq 0 \text {. }
$$

Then $t$ is a function of $t^{*}$ and $\left\|z^{*}\right\|=\|z\|<1$, and since $\operatorname{Re} c(t)>0$ for $t \geq 0$, we deduce that $\alpha(t) \geq 0$. Also $\alpha(t) \rightarrow \infty$ as $t \rightarrow \infty$ by the assumption (i). Moreover, it is clear that $\alpha(\cdot)$ is strictly increasing and continuous on $[0, \infty)$, and thus $\alpha(\cdot):[0, \infty) \rightarrow[0, \infty)$ is one-to-one.

Now let $h^{*}: B \times[0, \infty) \rightarrow X$ be given by

$$
h^{*}\left(z, t^{*}\right)=\frac{1}{\operatorname{Re} c(t)}\left[e^{i \beta(t)} h\left(e^{-i \beta(t)} z, t\right)-i \operatorname{Im} c(t) z\right] .
$$

Then $h^{*}\left(\cdot, t^{*}\right) \in H(B), h^{*}\left(0, t^{*}\right)=0$ and $D h^{*}\left(0, t^{*}\right)=I$ for $t^{*} \geq 0$. Moreover $h^{*}$ is continuous on $B \times[0, \infty)$, and

$$
\begin{gathered}
\operatorname{Re}\left[l_{z}\left(h^{*}\left(z, t^{*}\right)\right)\right]=\frac{1}{\operatorname{Re} c(t)} \operatorname{Re}\left[l_{z}\left(e^{i \beta(t)} h\left(z e^{-i \beta(t)}, t\right)\right)\right]= \\
=\frac{1}{\operatorname{Re} c(t)} \operatorname{Re}\left[l_{z e^{-i \beta(t)}} h\left(z e^{-i \beta(t)}, t\right)\right]>0, \quad z \in B \backslash\{0\}, t \geq 0,
\end{gathered}
$$

where we have used the fact that $l_{z e^{-i \beta(t)}}(\cdot)=e^{i \beta(t)} l_{z}(\cdot) \in T\left(z e^{-i \beta(t)}\right)$ for each $z \in B \backslash\{0\}$ and $t \geq 0$.

Hence in view of Lemma 1.5, we deduce that for each $s^{*} \geq 0$ and $z^{*} \in B$, the initial value problem

$$
\frac{\partial v^{*}}{\partial t^{*}}=-h^{*}\left(v^{*}, t^{*}\right), \quad t^{*} \geq s^{*}, v^{*}\left(s^{*}\right)=z^{*},
$$

has a unique solution $v^{*}\left(t^{*}\right)=v^{*}\left(z^{*}, s^{*}, t^{*}\right)=e^{s^{*}-t^{*}} z^{*}+\cdots$ such that for fixed $s^{*}$ and $t^{*}, v^{*}\left(\cdot, s^{*}, t^{*}\right)$ is a univalent Schwarz mapping. 
Further, let $v(z, s, t)=e^{-i \beta(t)} v^{*}\left(e^{i \beta(s)} z, \alpha(s), \alpha(t)\right)$ for $z \in B$ and $0 \leq s \leq t<\infty$. Then $v(\cdot, s, t) \in H(B), v(0, s, t)=0$ and $D v(0, s, t)=$ $e^{i(\beta(s)-\beta(t))} e^{s^{*}-t^{*}} I=a(s) / a(t) I$. Moreover $v(\cdot, s, t)$ is univalent on $B$ and

$$
\|v(z, s, t)\|<1, \quad t \geq s \geq 0, z \in B .
$$

Therefore $v(\cdot, s, t)$ is a univalent Schwarz mapping, and short computations based on (2.2) yield the following relation

$$
\frac{\partial v}{\partial t}(z, s, t)=-h(v(z, s, t), t), \quad t \geq s, v(z, s, s)=z .
$$

Hence $v(t)=v(z, s, t)$ is a solution of the initial value problem (2.1). Finally the uniqueness of solutions of the initial value problem (2.2) implies the uniqueness of solutions to (2.1). Indeed, if $u(t)=u(z, s, t)$ is another solution of the initial value problem (2.1), then $u^{*}(z, \alpha(s), \alpha(t))=e^{i \beta(t)} u\left(e^{-i \beta(s)} z, s, t\right)$ satisfies the initial value problem (2.2), and thus $u^{*}$ must be equal to $v^{*}$. Hence $u$ and $v$ are equal too, and this completes the proof.

Next, we prove that the solution of (2.1) generates Loewner chains (cf. [Por2, Theorems 2 and 3], [Por3, Lemma 4.5]; in the finite dimensional case compare with [Ch-Re, Theorem 2.1] and [Be, Lemma 1]).

Theorem 2.2. Let $h: B \times[0, \infty) \rightarrow X$ satisfy the assumptions $(i)$ and (ii) of Theorem 2.1. Also let $v(t)=v(z, s, t)$ be the solution of the initial value problem (2.1). Then there exists the limit

$$
\lim _{t \rightarrow \infty} a(t) v(z, s, t)=f(z, s)
$$

for all $z \in B$ and $s \geq 0$. The mapping $f(\cdot, s)$ is holomorphic and univalent on $B, f(0, s)=0, D f(0, s)=a(s) I, s \geq 0$, and $f(z, s)=f(v(z, s, t), t)$, $z \in B, t \geq s \geq 0$. Consequently, $f(z, t)$ is a Loewner chain. Moreover, $f(\cdot, s)$ satisfies the growth result

$$
\frac{|a(s)|\|z\|}{(1+\|z\|)^{2}} \leq\|f(z, s)\| \leq \frac{|a(s)|\|z\|}{(1-\|z\|)^{2}}, \quad z \in B, s \geq 0 .
$$

Further, if $f(z, \cdot)$ is differentiable on $[0, \infty)$ for each $z \in B$, then

$$
\left|\frac{1}{2} l_{w}\left(D^{2} f(0, s)(w, w)\right)\right| \leq 2|a(s)|, \quad\|w\|=1, \quad l_{w} \in T(w), \quad s \geq 0 .
$$

Proof. If $h^{*}\left(z, t^{*}\right)$ and $v^{*}\left(z, s^{*}, t^{*}\right)$ are the same mappings as in the proof of Theorem 2.1, we deduce in view of Lemma 1.5 that the limit

$$
\lim _{t^{*} \rightarrow \infty} e^{t^{*}} v^{*}\left(z, s^{*}, t^{*}\right)=f_{*}\left(z, s^{*}\right)
$$

exists for each $z \in B$ and $s^{*} \geq 0$, and $f_{*}\left(z, t^{*}\right)$ is a normalized Loewner chain. On the other hand, taking into account (1.4) we have

$$
\frac{e^{s^{*}}\|z\|}{(1+\|z\|)^{2}} \leq\left\|f_{*}\left(z, s^{*}\right)\right\| \leq \frac{e^{s^{*}}\|z\|}{(1-\|z\|)^{2}}, \quad z \in B, s^{*} \geq 0 .
$$


Further, let $f: B \times[0, \infty) \rightarrow X$ be given by

$$
f(z, t)=f_{*}\left(e^{i \beta(t)} z, \alpha(t)\right) \text { for } z \in B, t \geq 0,
$$

where $\alpha(t)$ and $\beta(t)$ are defined in the proof of Theorem 2.1. Then it is clear that $f(\cdot, t) \in H(B), f(\cdot, t)$ is univalent on $B, f(0, t)=0, D f(0, t)=a(t) I$, $t \geq 0$. On the other hand, if $w=w(z, s, t)$ is given by

$$
w(z, s, t)=e^{-i \beta(t)} v^{*}\left(e^{i \beta(s)} z, \alpha(s), \alpha(t)\right), z \in B, t \geq s \geq 0,
$$

then $w(\cdot, s, t)$ is a univalent Schwarz mapping and $f(z, s)=f(w(z, s, t), t)$ for $z \in B$ and $t \geq s \geq 0$. Therefore, $f(z, t)$ is a Loewner chain. Moreover, as in the proof of Theorem 2.1 we deduce that $w(t)=w(z, s, t)$ satisfies the initial value problem (2.1). Therefore, $w(z, s, t)=v(z, s, t)$ for $z \in B$ and $t \geq s \geq 0$.

On the other hand, the relation (2.6) yields that

$$
\begin{aligned}
\lim _{t \rightarrow \infty} a(t) v(z, s, t) & =\lim _{t \rightarrow \infty} e^{\alpha(t)} v^{*}\left(e^{i \beta(s)} z, \alpha(s), \alpha(t)\right) \\
& =f_{*}\left(e^{i \beta(s)} z, \alpha(s)\right)=f(z, s) .
\end{aligned}
$$

Hence (2.3) holds.

Next, from (2.7) we deduce that

$$
\frac{e^{\alpha(s)}\|z\|}{(1+\|z\|)^{2}} \leq\left\|f\left(e^{-i \beta(s)} z, s\right)\right\| \leq \frac{e^{\alpha(s)}\|z\|}{(1-\|z\|)^{2}},
$$

and since $|a(s)|=e^{\alpha(s)}, s \geq 0$, we obtain (2.4), as desired.

We omit the proof of the relation $(2.5)$, since it suffices to apply arguments similar to those in the proof of [Por1, Theorem 3] or [Gr-Ha-Ko, Theorem $2.14]$.

According to Theorem 2.2 we are now able to introduce the following definition (cf. [Por1], [Ko], [Gr-Ha-Ko]):

Definition 2.3. Let $f: B \rightarrow X$ be a normalized locally biholomorphic mapping. We say that $f$ has parametric representation if there is a mapping $h: B \times[0, \infty) \rightarrow X$, which satisfies the assumptions (i) and (ii) of Theorem 2.1, such that

$$
\lim _{t \rightarrow \infty} a(t) v(z, t)=f(z), \quad z \in B,
$$

where $v=v(z, t)$ is the unique solution of the initial value problem

$$
\frac{\partial v}{\partial t}=-h(v, t), \quad t \geq 0, v(z, 0)=z, z \in B .
$$

Further, we say that $f$ has normalized parametric representation if $a(t)=e^{t}$ for $t \geq 0$ (cf. [Por1], [Ko], [Gr-Ha-Ko]). Taking into account Theorem 2.2, we deduce that if $f$ has parametric representation (not necessarily normalized), then $f$ is biholomorphic on $B$. 
Let $S(B)$ be the set of normalized biholomorphic mappings on $B$ and $S^{0}(B)$ be the subset of $S(B)$ consisting of those mappings which have parametric representation on $B$. Also let $S^{1}(B)$ be the subset of $S(B)$ consisting of those mappings which can be embedded in Loewner chains (i.e. $f \in S^{1}(B)$ if $f$ is normalized biholomorphic on $B$ and there is a Loewner chain $f(z, t)$ such that $f(z, 0)=f(z), z \in B)$. Then it is clear that $S^{0}(B) \subseteq S^{1}(B)$ and we shall show that $S^{0}(B) \subset S^{1}(B)$ and respectively $S^{0}(B) \subset S(B)$ (cf. [Ko], [Gr-Ha-Ko]).

In the next result we prove that solutions of the Loewner differential equation give rise to Loewner chains (compare with [Por2, Theorem 6], [Pf, Theorem 2.3]; cf. [Cu, Theorem 2] and [Ch-Re, Theorem 2.1] when $\left.X=\mathbb{C}^{n}\right)$.

Theorem 2.4. Let $f: B \times[0, \infty) \rightarrow X$ be a continuous mapping such that $f(\cdot, t) \in H(B), f(0, t)=0, D f(0, t)=a(t) I, t \geq 0$, where $a(\cdot):[0, \infty) \rightarrow \mathbb{C}$ is a function of class $C^{1}$ on $[0, \infty), a(t) \neq 0, t \geq 0,|a(\cdot)|$ is strictly increasing on $[0, \infty)$ and $a(t) \rightarrow \infty$ as $t \rightarrow \infty$. Also assume that $f(z, \cdot)$ is differentiable on $[0, \infty)$ for each $z \in B$, and there exist $r \in(0,1), t_{0}>0$, and $M>0$ such that

$$
\|f(z, t)\| \leq M|a(t)|, \quad\|z\| \leq r, t \geq t_{0} .
$$

Further, assume that there exists a mapping $h=h(z, t)=c(t) z+\cdots$, which satisfies the conditions $(i)$ and (ii) of Theorem 2.1, where $c(t)=$ $a^{\prime}(t) / a(t), t \geq 0$, and that

$$
\frac{\partial f}{\partial t}(z, t)=D f(z, t) h(z, t), \quad z \in B, t \geq 0 .
$$

Then $f(z, t)$ is a Loewner chain which satisfies the relation (2.3). Hence, if $f(z)=f(z, 0)$ is locally biholomorphic on $B$ then $f$ has parametric representation.

Proof. Let

$$
t^{*}=\log \left|\frac{a(t)}{a(0)}\right| \text { and } \theta(t)=\arg \left[\frac{a(t)}{a(0)}\right], t \geq 0 .
$$

Also let

$$
f_{*}\left(z, t^{*}\right)=\frac{1}{a(0)} f\left(z e^{-i \theta(t)}, t\right)
$$

Then $f_{*}\left(\cdot, t^{*}\right) \in H(B), f_{*}\left(0, t^{*}\right)=0$ and $D f_{*}\left(0, t^{*}\right)=e^{t^{*}} I$ for $t^{*} \geq 0$. Also it is clear that $f_{*}(z, \cdot)$ is differentiable on $[0, \infty)$ for each $z \in B$, and $f_{*}$ is continuous on $B \times[0, \infty)$.

Obviously,

$$
\frac{d t^{*}}{d t}=\operatorname{Re}\left[\frac{a^{\prime}(t)}{a(t)}\right] \text { and } \frac{d \theta}{d t}(t)=\operatorname{Im}\left[\frac{a^{\prime}(t)}{a(t)}\right], t \geq 0 .
$$


Taking into account these relations and (2.9), we easily obtain

$$
\frac{\partial f_{*}}{\partial t^{*}}\left(z, t^{*}\right)=D f_{*}\left(z, t^{*}\right) h_{*}\left(z, t^{*}\right), \quad z \in B, t^{*} \geq 0,
$$

where

$$
h_{*}\left(z, t^{*}\right)=\frac{1}{\operatorname{Re}\left[\frac{a^{\prime}(t)}{a(t)}\right]}\left\{e^{i \theta(t)} h\left(z e^{-i \theta(t)}, t\right)-i \operatorname{Im}\left[\frac{a^{\prime}(t)}{a(t)}\right] z\right\} .
$$

As in the proof of Theorem 2.1 we deduce that $h_{*}\left(\cdot, t^{*}\right) \in H(B)$, $h_{*}\left(0, t^{*}\right)=0, D h_{*}\left(0, t^{*}\right)=I$ and $\operatorname{Re}\left[l_{z}\left(h_{*}\left(z, t^{*}\right)\right)\right]>0$ for $z \in B \backslash\{0\}$ and $t^{*} \geq 0$. Since $h(z, t)$ satisfies the assumption (ii) of Theorem 2.1 and $a(\cdot)$ is of class $C^{1}$ on $[0, \infty)$, we see that $h_{*}$ is continuous on $B \times[0, \infty)$.

On the other hand, using the relation (2.8), we deduce that

$$
\left\|f_{*}\left(z, t^{*}\right)\right\| \leq M e^{t^{*}}, \quad\|z\| \leq r, \quad t^{*} \geq t_{0}^{*} .
$$

In view of Lemma 1.6 we conclude that $f_{*}\left(z, t^{*}\right)$ is a Loewner chain, and for all $z \in B$ and $s \geq 0$, the limit

$$
\lim _{t^{*} \rightarrow \infty} e^{t^{*}} v_{*}\left(z, s^{*}, t^{*}\right)=f_{*}\left(z, s^{*}\right)
$$

exists, where $v_{*}\left(t^{*}\right)=v_{*}\left(z, s^{*}, t^{*}\right)$ is the unique solution of the initial value problem

$$
\frac{\partial v_{*}}{\partial t^{*}}=-h_{*}\left(v_{*}, t^{*}\right), \quad t^{*} \geq s^{*}, v_{*}\left(s^{*}\right)=z .
$$

Using arguments similar to those in the proofs of Theorems 2.1 and 2.2, we deduce that $v(z, s, t)=e^{-i \theta(t)} v_{*}\left(z e^{i \theta(s)}, s^{*}, t^{*}\right)$ is the unique solution of the initial value problem

$$
\frac{\partial v}{\partial t}=-h(v, t), \quad t \geq s, v(z, s, s)=z,
$$

and further, $f(z, s)=f(v(z, s, t), t)$ for $z \in B$ and $t \geq s \geq 0$. Also since $f_{*}\left(\cdot, t^{*}\right)$ is univalent on $B$, it follows that $f(\cdot, t)$ is also univalent on $B$. Finally, in view of the relation $(2.10)$, we conclude that the limit

$$
\lim _{t \rightarrow \infty} a(t) v(z, s, t)=f(z, s)
$$

exists for each $z \in B$ and $s \geq 0$. This completes the proof.

REMARK 2.5. Let $f$ be a normalized locally biholomorphic mapping. In view of Theorem 2.2 and Definition 2.3 we deduce that if $f$ has parametric representation, then there is a Loewner chain $f(z, t)$ such that $f(z)=f(z, 0)$, $z \in B$. This chain satisfies the following conditions: $f(0, t)=0, \operatorname{Df}(0, t)=$ $a(t) I, t \geq 0$, where $a(\cdot)$ is a function of class $C^{1}$ on $[0, \infty), a(0)=1,|a(\cdot)|$ is strictly increasing on $[0, \infty),|a(t)| \rightarrow \infty$ as $t \rightarrow \infty$, and for each $r \in(0,1)$ there is a positive number $M=M(r)>0$ such that $\|f(z, t)\| \leq M|a(t)|$ for $t \geq 0$ and $\|z\| \leq r$. 
In the finite dimensional case, Graham, Hamada and Kohr [Gr-Ha-Ko, Theorems 1.4 and 1.10] have recently proved that a normalized holomorphic mapping $f$ has normalized parametric representation if and only if there is a normalized Loewner chain $f(z, t)$ such that $\left\{e^{-t} f(z, t)\right\}_{t \geq 0}$ is a normal family on $B, f(z, \cdot)$ is locally Lipschitz continuous on $[0, \infty)$ locally uniformly with respect to $z \in B$, and such that $f(z)=f(z, 0), z \in B$.

Taking into account Theorem 2.4 and Remark 2.5, we conjecture the following result in infinite dimensions:

Conjecture 2.6. Let $f: B \rightarrow X$ be a normalized locally biholomorphic mapping. Then $f$ has parametric representation if and only if there is a Loewner chain $f(z, t)$ such that $f(z)=f(z, 0), z \in B$, and this chain satisfies the following conditions:

(i) $f(0, t)=0, D f(0, t)=a(t) I$, where $a(\cdot)$ is a function of class $C^{1}$ on $[0, \infty), a(0)=1,|a(\cdot)|$ is strictly increasing on $[0, \infty)$, and $|a(t)| \rightarrow \infty$ as $t \rightarrow \infty$;

(ii) $f(z, \cdot)$ is locally Lipschitz continuous on $[0, \infty)$ uniformly with respect to $z \in \bar{B}_{r}, r \in(0,1)$;

(iii) there are $r \in(0,1), t_{0}>0$ and $M>0$ such that

$$
\|f(z, t)\| \leq M|a(t)|, \quad\|z\| \leq r, \quad t \geq t_{0} .
$$

We conclude this section with some examples which show that in infinite dimensions the following inclusion relations

$$
S^{0}(B) \subseteq S(B) \text { and } S^{0}(B) \subseteq S^{1}(B)
$$

are strict (cf. [Gr-Ha-Ko]).

Example 2.7. Let $X=\ell_{2}=\left\{z=\left(z_{1}, z_{2}, \ldots\right):\|z\|^{2}=\sum_{j=1}^{\infty}\left|z_{j}\right|^{2}<\infty\right\}$ and let $f: B \rightarrow X$ be given by

$$
f(z)=\left(z_{1}+a z_{2}^{2}, z_{2}, z_{3}, \ldots, z_{n}, \ldots\right), \quad z=\left(z_{1}, z_{2}, \ldots, z_{n}, \ldots\right) \in B,
$$

where $a \in \mathbb{C},|a|>4 \sqrt{2}$. It is obvious that $f \in S(B)$.

Let $l_{z}(w)=\frac{1}{\|z\|} \sum_{j=1}^{\infty} z_{j} \bar{w}_{j}$ for $z \neq 0$ and $w=\left(w_{1}, w_{2}, \ldots\right) \in X$. Then $l_{z} \in T(z)$ and a short computation yields that

$$
\left|\frac{1}{2} l_{z}\left(D^{2} f(0)(z, z)\right)\right|=|a| \cdot\left|z_{1} z_{2}^{2}\right|=\frac{|a|}{2 \sqrt{2}}>2
$$

for $z=(r, r, 0, \ldots, 0, \ldots) \in X$ and $r=1 / \sqrt{2}$. From (2.5) we deduce that $f \notin S^{0}(B)$. 
ExAmple 2.8. Again let $X=\ell_{2}$ and consider the Loewner chain

$$
f(z, t)=\left(\frac{a(t) z_{1}}{\left(1-z_{1}\right)^{2}}, \frac{a(t) z_{2}}{\left(1-z_{2}\right)^{2}}, \ldots\right), \quad z=\left(z_{1}, z_{2}, \ldots\right) \in B, t \geq 0,
$$

where $a(\cdot):[0, \infty) \rightarrow \mathbb{C}$ is a function of class $C^{1}$ such that $a(0)=1,|a(\cdot)|$ is strictly increasing on $[0, \infty)$ and $a(t) \rightarrow \infty$ as $t \rightarrow \infty$.

Also let $A: X \rightarrow X$ be a normalized biholomorphic mapping, not the identity map, such that $\left\|A\left(z_{0}\right)\right\|>\left\|z_{0}\right\|$ for some point $z_{0} \in X$. Such a map always exists on $\ell_{2}$ (otherwise, $A$ should be the identity by [Fr-Ve, Theorem 2.3.9]) and after rotation with unitary transformations, we may assume that there is $\rho>0$ such that $\|A(\rho, 0, \ldots)\|>\rho$. Next, choose $r \in(0,1)$ such that $r /(1-r)^{2}=\rho$. It is not difficult to show that $(A \circ f)(z, t)$ is a Loewner chain, thus $A \circ f \in S^{1}(B)$, where $f(z)=f(z, 0), z \in B$. Moreover,

$$
\begin{aligned}
\|(A \circ f)(r, 0,0, \ldots)\| & =\left\|A\left(\frac{r}{(1-r)^{2}}, 0, \ldots\right)\right\|=\|A(\rho, 0, \ldots)\| \\
& >\rho=\frac{r}{(1-r)^{2}} .
\end{aligned}
$$

In view of the relation (2.4), we deduce that $A \circ f \notin S^{0}(B)$.

\section{UNIVALENCE CRITERIA}

In this section we shall assume that $X$ is a complex Hilbert space with inner product $\langle\cdot, \cdot\rangle$ and $B$ is the unit ball of $X$. We next apply the result of Theorem 2.4 to obtain some univalence criteria on $B$. We begin with a generalization to infinite dimensions of a result due to Brodskii $[\mathrm{Br}]$.

Theorem 3.1. Let $c \in \mathbb{C},|c|<1$, and let $f: B \rightarrow X$ be a normalized locally biholomorphic mapping such that

$$
\|(1+c) D f(z)-I\|<1, \quad z \in B .
$$

Then $f$ has parametric representation on $B$, and thus is biholomorphic on $B$.

Proof. Let

$$
f(z, t)=f\left(z e^{-t}\right)+\frac{\left(e^{t}-e^{-t}\right) z}{1+c}, \quad z \in B, t \geq 0 .
$$

We prove that $f(z, t)$ satisfies the assumptions of Theorem 2.4.

Clearly $f(z, \cdot)$ is of class $C^{\infty}$ on $[0, \infty)$ for $z \in B, f(\cdot, t) \in H(B), f(0, t)=$ $0, D f(0, t)=a(t) I$, where $a(t)=\left(e^{t}+c e^{-t}\right) /(1+c)$ for $t \geq 0$. Also it is easy to see that $|a(\cdot)|$ is strictly increasing on $[0, \infty), a(t) \rightarrow \infty$ as $t \rightarrow \infty$, and since $a(0)=1$ it follows that $a(t) \neq 0, t \geq 0$.

Moreover, since $f$ is continuous on $B$, it is bounded in a neighbourhood of 0 , and thus there exist $r \in(0,1)$ and $M=M(r)>0$ such that $\left\|f\left(z e^{-t}\right)\right\| \leq M$ for $\|z\| \leq r$ and $t \geq 0$. Since

$$
e^{t}-e^{-t} \leq\left|e^{t}+c e^{-t}\right|, \quad t \geq 0,
$$


it is easy to see that there exists a positive number $K=K(r)$ such that

$$
\|f(z, t)\| \leq K|a(t)|, \quad\|z\| \leq r, t \geq 0 .
$$

Indeed, since $|a(t)| \geq 1$ for $t \geq 0$, we deduce that

$$
\begin{gathered}
\|f(z, t)\| \leq M|a(t)|+\frac{e^{t}-e^{-t}}{|1+c|} r \\
\leq M|a(t)|+|a(t)| r=(M+r)|a(t)|=K|a(t)|, \quad\|z\| \leq r, t \geq 0 .
\end{gathered}
$$

On the other hand, straightforward computations yield the following relations

and

$$
D f(z, t)=\frac{e^{t}}{1+c}[I-E(z, t)]
$$

$$
\frac{\partial f}{\partial t}(z, t)=\frac{e^{t}}{1+c}[I+E(z, t)](z)
$$

where

$$
E(z, t)=e^{-2 t}\left[I-(1+c) D f\left(z e^{-t}\right)\right], \quad z \in B, t \geq 0 .
$$

Using the hypothesis, we see that $\|E(z, t)\|<1, z \in B, t \geq 0$, and hence $I-E(z, t)$ is an invertible linear operator.

Letting $h(z, t)=[I-E(z, t)]^{-1}[I+E(z, t)](z)$, we deduce that $h(\cdot, t) \in$ $H(B), h(z, t)$ is continuous in $(z, t) \in B \times[0, \infty), h(0, t)=0$, and

$$
\begin{aligned}
\|h(z, t)-z\| & =\|E(z, t)[h(z, t)+z]\| \leq\|E(z, t)\| \cdot\|h(z, t)+z\|< \\
& <\|h(z, t)+z\|, \quad z \in B, t \in[0, \infty) .
\end{aligned}
$$

Therefore $\|h(z, t)-z\|<\|h(z, t)+z\|$ and this inequality implies that $\operatorname{Re}\langle h(z, t), z\rangle>0$ for $z \in B \backslash\{0\}, t \geq 0$. Since $D h(0, t)=\left[\left(e^{t}-\right.\right.$ $\left.\left.c e^{-t}\right) /\left(e^{t}+c e^{-t}\right)\right] I$, we deduce that $c(t)=\left[\left(e^{t}-c e^{-t}\right) /\left(e^{t}+c e^{-t}\right)\right]$ is a continuous function on $[0, \infty)$ and it is obvious that $c(t)=a^{\prime}(t) / a(t)$ for $t \geq 0$. On the other hand, since

$$
\begin{aligned}
\int_{0}^{\infty} \operatorname{Re} c(t) d t & =\int_{0}^{\infty} \operatorname{Re} \frac{a^{\prime}(t)}{a(t)} d t=\lim _{t \rightarrow \infty} \int_{0}^{t} \frac{d}{d \tau} \operatorname{Re}[\log a(\tau)] d \tau \\
& =\lim _{t \rightarrow \infty} \log |a(t)|=\infty
\end{aligned}
$$

we deduce that $h(z, t)$ satisfies the assumptions of Theorem 2.1.

Further, using the above arguments, we conclude that $f(z, t)$ satisfies the differential equation

$$
\frac{\partial f}{\partial t}(z, t)=D f(z, t) h(z, t), \quad z \in B, t \in[0, \infty) .
$$

Hence, in view of Theorem 2.4 we deduce that $f(z, t)$ is a Loewner chain, and for each $s \geq 0$ and $z \in B$,

$$
f(z, s)=\lim _{t \rightarrow \infty} a(t) v(z, s, t),
$$


where $v(t)=v(z, s, t)$ is the solution of the initial value problem

$$
\frac{\partial v}{\partial t}=-h(v, t), \quad t \geq s, v(s)=z .
$$

Therefore $f(z)=f(z, 0)$ has parametric representation on $B$, and thus is biholomorphic, as desired. This completes the proof.

We next obtain a generalization to infinite dimensional complex Hilbert spaces of a result due to Ren and Ma [Re-Ma].

TheOREM 3.2. Let $f: B \rightarrow X$ be a normalized locally biholomorphic mapping and let $c \in \mathbb{C},|c|<1$. Also let $G: B \rightarrow L(X, X)$ be a mapping such that $G(0)=I, G$ is holomorphic on $B$ and $G(z)$ is invertible for each $z \in B$. Also assume the following conditions hold:

(i) $\left\|(1+c)[G(z)]^{-1} \operatorname{Df}(z)-I\right\|<1, z \in B$,

(ii) \|\|$z\left\|^{2}\left\{(1+c)[G(z)]^{-1} D f(z)-I\right\}+\left(1-\|z\|^{2}\right)[G(z)]^{-1} D G(z)(z, \cdot)\right\| \leq 1$, for all $z \in B$. Then $f$ is biholomorphic on $B$ and moreover, $f$ has parametric representation.

Proof. We only sketch some ideas of the proof, since it suffices to use arguments similar to those in the proof of Theorem 3.1. To this end, it suffices to consider the mapping

$$
f(z, t)=f\left(z e^{-t}\right)+\frac{\left(e^{t}-e^{-t}\right) G\left(z e^{-t}\right)(z)}{1+c}, \quad z \in B, t \geq 0 .
$$

This mapping satisfies the assumptions of Theorem 2.4. Indeed, $f(\cdot, t) \in$ $H(B), f(0, t)=0, D f(0, t)=a(t) I, t \geq 0$, where $a(t)=\left(e^{t}+c e^{-t}\right) /(1+c)$. By the proof of Theorem 3.1, we obtain that $|a(\cdot)|$ is strictly increasing on $[0, \infty), a(t) \neq 0, t \geq 0$, and $|a(t)| \rightarrow \infty$ as $t \rightarrow \infty$. On the other hand, it is not difficult to deduce that $f(z, t)$ satisfies the differential equation

$$
\frac{\partial f}{\partial t}(z, t)=D f(z, t) h(z, t), \quad z \in B, t \geq 0,
$$

where $h(z, t)=[I-E(z, t)]^{-1}[I+E(z, t)](z)$, and

$$
\begin{aligned}
E(z, t)= & -e^{-2 t}\left\{(1+c)\left[G\left(z e^{-t}\right)\right]^{-1} D f\left(z e^{-t}\right)-I\right\} \\
& -\left(1-e^{-2 t}\right)\left[G\left(z e^{-t}\right)\right]^{-1} D G\left(z e^{-t}\right)\left(z e^{-t}, \cdot\right),
\end{aligned}
$$

for all $z \in B$ and $t \geq 0$. Using the condition (i), we see that

$$
\|E(z, 0)\|=\left\|(1+c)[G(z)]^{-1} D f(z)-I\right\|<1, \quad z \in B .
$$

Also, using the condition (ii), we deduce for $t>0$ that

$$
\begin{aligned}
\sup _{\|w\|=1}\|E(w, t)\|= & \sup _{\|w\|=1}\|\| w e^{-t} \|^{2}\left\{(1+c)\left[G\left(w e^{-t}\right)\right]^{-1} D f\left(w e^{-t}\right)-I\right\} \\
& +\left(1-\left\|w e^{-t}\right\|^{2}\right)\left[G\left(w e^{-t}\right)\right]^{-1} D G\left(w e^{-t}\right)\left(w e^{-t}, \cdot\right) \| \leq 1 .
\end{aligned}
$$


Hence, in view of the maximum modulus theorem for holomorphic mappings into complex Banach spaces (see [Hi-Ph]), we deduce that $\|E(z, t)\|<1$ for $z \in B$. Thus $I-E(z, t)$ is an invertible linear operator for $z \in B$ and $t \geq 0$, and $h(z, t)$ is well defined and is holomorphic with respect to $z \in B$. On the other hand, $h(z, t)$ is continuous in $(z, t) \in B \times[0, \infty), h(0, t)=0$ and

$$
D h(0, t)=\frac{e^{t}-c e^{-t}}{e^{t}+c e^{-t}} I, \quad t \geq 0 .
$$

Moreover, as in the proof of Theorem 3.1, we deduce that $\operatorname{Re}\langle h(z, t), z\rangle>$ 0 for $z \in B \backslash\{0\}$ and $t \geq 0$. Therefore $h(z, t)$ satisfies the assumptions of Theorem 2.1. Finally, using the continuity of $f$ and $G$ near 0 , and an argument similar to that in the proof of Theorem 3.1, it is not difficult to deduce that there exist some numbers $r \in(0,1)$ and $K=K(r)>0$ such that

$$
\|f(z, t)\| \leq K|a(t)|, \quad\|z\| \leq r, \quad t \geq 0 .
$$

Hence taking into account Theorem 2.4, we deduce that $f(z, t)$ is a Loewner chain, and since $f(z)=f(z, 0)$ is locally biholomorphic on $B$, we conclude that $f$ has parametric representation, as desired. This completes the proof.

When $G(z)=D f(z), z \in B$, and $c=0$ we obtain an infinite dimensional version of [Pf, Theorem 2.4].

Corollary 3.3. Let $f: B \rightarrow X$ be a normalized locally biholomorphic mapping such that

$$
\left(1-\|z\|^{2}\right)\left\|[D f(z)]^{-1} D^{2} f(z)(z, \cdot)\right\| \leq 1, \quad z \in B .
$$

Then $f$ has parametric representation on $B$, and thus is biholomorphic on $B$.

On the other hand, letting $G(z)=D f(z), z \in B$, and $|c|<1$ in Theorem 3.2 , we obtain an infinite dimensional version of a result obtained in [Ch-Re] and $[\mathrm{Cu}]$.

Corollary 3.4. Let $f: B \rightarrow X$ be a normalized locally biholomorphic mapping and $c \in \mathbb{C}$ such that $|c|<1$. Assume that

$$
\|c\| z\left\|^{2} I+\left(1-\|z\|^{2}\right)[D f(z)]^{-1} D^{2} f(z)(z, \cdot)\right\| \leq 1, \quad z \in B .
$$

Then $f$ has parametric representation on $B$, and thus is biholomorphic on $B$.

\section{Spirallikeness of type $\alpha$ And ClOSE-TO-STARlikeness in Banach SPACES}

In this section we show that spirallikeness of type $\alpha$ can be characterized in terms of Loewner chains (cf. [Ha-Ko1, Theorem 3.1], [Pf-Su1]). We have 
Theorem 4.1. Let $\alpha \in(-\pi / 2, \pi / 2)$ and $f: B \rightarrow X$ be a normalized locally biholomorphic mapping. Then $f$ is spirallike of type $\alpha$ if and only if $f(z, t)=e^{(1-i a) t} f\left(e^{i a t} z\right)$ is a Loewner chain, where $a=\tan \alpha$. Hence $f$ is starlike if and only if $f(z, t)=e^{t} f(z)$ is a Loewner chain.

Proof. First assume that $f(z, t)$ is a Loewner chain. Then $f(z)=f(z, 0)$ is univalent on $B$ and since $f$ is locally biholomorphic, we deduce that $f$ is biholomorphic on $B$. Also if $v=v(z, s, t)$ is the transition mapping associated to $f(z, t)$, i.e.

$$
f(z, s)=f(v(z, s, t), t), \quad z \in B, t \geq s \geq 0,
$$

then it is easy to see that

$$
v(z, s, t)=e^{-i a t} f^{-1}\left(e^{(1-i a)(s-t)} f\left(e^{i a s} z\right)\right) .
$$

Thus $v(z, s, t)$ is of class $C^{\infty}$ with respect to $t$ for all $z \in B, s \geq 0$, and $t \geq s$, and the derivative $\partial v / \partial t$ is obviously holomorphic on $B$. Further, since $f(z, t)$ is of class $C^{\infty}$ on $B \times[0, \infty)$, we deduce in view of Lemma 1.7 that there is a mapping $h=h(z, t)$ such that $h(\cdot, t) \in \mathcal{M}$ and that

$$
\frac{\partial f}{\partial t}(z, t)=D f(z, t) h(z, t), \quad z \in B, t \geq 0 .
$$

A straightforward computation yields that $h(z, t)$ is given by

$$
h(z, t)=i a z+(1-i a) e^{-i a t}\left[D f\left(z e^{i a t}\right)\right]^{-1} f\left(e^{i a t} z\right) .
$$

Since $\operatorname{Re}\left[l_{z}(h(z, t))\right]>0$ for $z \in B \backslash\{0\}$ and $t \geq 0$, we deduce for $t=0$ that

$$
\operatorname{Re}\left[e^{-i \alpha} l_{z}\left([D f(z)]^{-1} f(z)\right)\right]>0, \quad z \in B \backslash\{0\},
$$

and hence $f$ is spirallike of type $\alpha$.

Conversely, assume $f$ is spirallike of type $\alpha$. Then $f(\cdot, t)$ is locally biholomorphic on $B, f(0, t)=0, D f(0, t)=e^{t} I, t \geq 0$, and $f(z, \cdot) \in C^{\infty}([0, \infty))$ for each $z \in B$. Also since $f$ is continuous at $z=0$, we deduce that there exist some numbers $r \in(0,1)$ and $K=K(r)>0$ such that

$$
\|f(z, t)\| \leq K e^{t} \text { for }\|z\| \leq r \text { and } t \geq 0 .
$$

Moreover, reversing the argument in the first step, we deduce that $f(z, t)$ satisfies the differential equation (4.1) with $h(z, t)$ given by $(4.2)$. Then $h(\cdot, t)$ is a normalized holomorphic mapping on $B$ and $h(z, t)$ is continuous with respect to $(z, t) \in B \times[0, \infty)$. Since $f$ is spirallike of type $\alpha$, we obtain

$$
\operatorname{Re}\left[l_{z}(h(z, t))\right]=\frac{1}{\cos \alpha} \operatorname{Re}\left[e^{-i \alpha} l_{z e^{i a t}}\left(\left[D f\left(z e^{i a t}\right)\right]^{-1} f\left(z e^{i a t}\right)\right)\right]>0,
$$

for $z \in B \backslash\{0\}$ and $t \geq 0$. Therefore $h(\cdot, t) \in \mathcal{M}, t \geq 0$. Taking into account Theorem 2.4, we deduce that $f(z, t)$ is a Loewner chain, and moreover $f(z)=f(z, 0)$ has parametric representation on $B$. This completes the proof. 
REMARK 4.2. Taking into account the proof of Theorem 4.1, we deduce that if $f$ is normalized starlike or spirallike of type $\alpha \in(-\pi / 2, \pi / 2)$, then $f$ has parametric representation on $B$. The same argument applies to close-tostarlike mappings (see also [Ko], [Gr-Ha-Ko]).

The following result provides a sufficient condition for close-to-starlikeness on the unit ball of $X$ in some special case. To this end, let $H(B, \mathbb{C})$ be the set of holomorphic functions from $B$ into $\mathbb{C}$.

THEOREM 4.3. Let $\phi, \psi \in H(B, \mathbb{C})$ be two functions such that $\phi(z) \neq 0$ for $z \in B \backslash\{0\}$ and $\phi(0)=\psi(0)=1$. Let $f(z)=z \phi(z)$ and $g(z)=z \psi(z)$, and assume that $f$ is locally biholomorphic on $B$ and $g$ is starlike. Also assume that $f(\cdot, t)$ is locally biholomorphic on $B$, where $f(z, t)$ is given by $(1.1)$, and

$$
\operatorname{Re}\left[l_{z}\left([D f(z)]^{-1} g(z)\right)\right]>0, \quad z \in B \backslash\{0\}, l_{z} \in T(z) .
$$

Then $f$ is close-to-starlike with respect to $g$.

Proof. In view of [Pf-Su2, Theorem 2] the condition of starlikeness of $g$ is equivalent to

$$
\operatorname{Re}\left[1+\frac{D \psi(z)(z)}{\psi(z)}\right]>0, \quad z \in B
$$

On the other hand, an argument similar to that in the proof of [Pf-Su2, Theorem 2] yields that (4.3) is equivalent to the following relation

$$
\operatorname{Re}\left[\frac{\phi(z)+D \phi(z)(z)}{\psi(z)}\right]>0, \quad z \in B .
$$

Hence

$$
\operatorname{Re}\left[e^{-t} \frac{\phi(z)+D \phi(z)(z)}{\psi(z)}+\left(1-e^{-t}\right) \frac{\psi(z)+D \psi(z)(z)}{\psi(z)}\right]>0
$$

for all $z \in B$ and $t \geq 0$. Next, let $h: B \times[0, \infty) \rightarrow X$ be given by

$h(z, t)=z\left[e^{-t} \frac{\phi(z)+D \phi(z)(z)}{\psi(z)}+\left(1-e^{-t}\right) \frac{\psi(z)+D \psi(z)(z)}{\psi(z)}\right]^{-1}, z \in B, t \geq 0$.

Then $h(\cdot, t) \in \mathcal{M}, h(z, t)$ is continuous in $(z, t) \in B \times[0, \infty)$, and it is not difficult to deduce that

$$
\frac{\partial f}{\partial t}(z, t)=D f(z, t) h(z, t), \quad z \in B, t \geq 0 .
$$

Recall that $f(z, t)=f(z)+\left(e^{t}-1\right) g(z), z \in B, t \geq 0$.

On the other hand, it is obvious that there are $r \in(0,1)$ and $K=K(r)>$ 0 such that

$$
\|f(z, t)\| \leq K(r) e^{t}, \quad\|z\| \leq r, \quad t \geq 0
$$

in view of the continuity of $f$ and $g$ at the origin. Therefore, taking into account Lemma 1.6, we deduce that $f(z, t)$ is a Loewner chain. Finally since 
$f(\cdot, t)$ is locally biholomorphic on $B$, we conclude that $f(z)=f(z, 0)$ is closeto-starlike. This completes the proof.

We close this section with an example, which is a direct application of Theorem 4.3.

EXAMPLE 4.4. Let $X=\ell_{2}$ and let $f_{1}$ be a normalized close-to-convex function with respect to a normalized convex function $g_{1}$ on the unit disc $U$. Also let $\psi(z)=g_{1}^{\prime}\left(z_{1}\right)$ and $\phi(z)=f_{1}\left(z_{1}\right) / z_{1}$ for $z=\left(z_{1}, z_{2}, \ldots\right) \in B$. Since $g_{1}$ is convex on $U$, the relation (4.4) holds, and hence $g(z)=z \psi(z)$ is starlike. Let $f_{1}\left(z_{1}, t\right)=f_{1}\left(z_{1}\right)+\left(e^{t}-1\right) z_{1} g_{1}^{\prime}\left(z_{1}\right)$ for $t \geq 0$. Then $f_{1}\left(z_{1}, t\right)$ is a normalized Loewner chain on $U$. Since

$$
D f(z, t)=\frac{f_{1}\left(z_{1}, t\right)}{z_{1}}\left(I+z \frac{z_{1} f_{1}^{\prime}\left(z_{1}, t\right)-f_{1}\left(z_{1}, t\right)}{z_{1} f_{1}\left(z_{1}, t\right)}\left\langle\cdot, e_{1}\right\rangle\right),
$$

it follows that $[D f(z, t)]^{-1}$ exists if and only if $f_{1}\left(z_{1}, t\right) / z_{1} \neq 0$ and

$$
1+\frac{z_{1} f_{1}^{\prime}\left(z_{1}, t\right)-f_{1}\left(z_{1}, t\right)}{f_{1}\left(z_{1}, t\right)} \neq 0
$$

for $\left|z_{1}\right|<1$ and $t \geq 0$. Since $f_{1}(\cdot, t)$ is a normalized Loewner chain on $U$, these conditions are satisfied. Thus $[D f(z, t)]^{-1}$ exists, and hence $f(\cdot, t)$ is locally biholomorphic on $B$ for each $t \geq 0$. Finally, since $f_{1}$ is close-toconvex with respect to $g_{1}$, the relation (4.5) holds, and hence the relation (4.3) holds. Consequently, $f(z)=z f_{1}\left(z_{1}\right) / z_{1}$ is close-to-starlike with respect to $g(z)=z g_{1}^{\prime}\left(z_{1}\right)$.

\section{REFERENCES}

[Be] J. Becker, Über die Lösungsstruktur einer differentialgleichung in der konformen Abbildung, J. Reine Angew. Math., 285 (1976), 66-74.

[Br] A.A. Brodskii, Quasiconformal extension of biholomorphic mappings, Theory of Mappings and Approximation of Functions (G. Suvarov, Ed.), pp. 30-34, Naukova Dumka, Kiev, 1983.

[Ch-Re] H.B. Chen and F. Ren, Univalence of holomorphic mappings and growth theorems for close-to starlike mappings in finitely dimensional Banach spaces, Acta Math. Sinica (N.S.) 10 (1994), Special Issue, 207-214.

[Cu] P. Curt, A generalization in n-dimensional complex space of Ahlfors and Becker's criterion for univalence, Studia Univ. Babeş-Bolyai (Mathematica), 39 (1994), 31-38.

[Fr-Ve] T. Franzoni and E. Vesentini, Holomorphic Maps and Invariant Distances, NorthHolland Math. Studies, 1980.

[Gr-Ha-Ko] I. Graham, H. Hamada and G. Kohr, Parametric representation of univalent mappings in several complex variables, Canadian J. Math. 54 (2002), 324-351.

[Ha-Ko1] H. Hamada and G. Kohr, Subordination chains and the growth theorem of spirallike mappings, Mathematica (Cluj), 42(65) (2000), 153-161.

[Ha-Ko2] H. Hamada and G. Kohr, $\Phi$-like and convex mappings in infinite dimensional spaces, Rev. Roum. Math. Pures Appl., 47 (2002), 315-328.

[Hi-Ph] E. Hille and R.S. Phillips, Functional Analysis and Semigroups, Amer. Math. Soc. Coll. Publ. 31, Providence, R.I., 1957. 
[Ko] G. Kohr, Using the method of Löwner chains to introduce some subclasses of biholomorphic mappings in $\mathbb{C}^{n}$, Rev. Roum. Math. Pures Appl. 46 (2001), 743-760.

[Pf] J.A. Pfaltzgraff, Subordination chains and univalence of holomorphic mappings in $\mathbb{C}^{n}$, Math. Ann. 210 (1974), 55-68.

[Pf-Su1] J.A. Pfaltzgraff, T.J. Suffridge, Close-to-starlike holomorphic functions of several variables, Pacif. J. Math. 57 (1975), 271-279.

[Pf-Su2] J.A. Pfaltzgraff, T.J. Suffridge, An extension theorem and linear invariant families generated by starlike maps, Ann. Univ. Mariae Curie Sklodowska, Sect.A 53 (1999), 193-207.

[Po] C. Pommerenke, Univalent Functions, Vandehoeck \& Ruprecht, Göttingen, 1975.

[Por1] T. Poreda, On the univalent holomorphic maps of the unit polydisc in $\mathbb{C}^{n}$ which have the parametric representation, I - the geometrical properties, Ann. Univ. Mariae Curie Sklodowska, Sect A 41 (1987), 105-113.

[Por2] T. Poreda, On the univalent subordination chains of holomorphic mappings in Banach spaces, Commentationes Math., 28 (1989), 295-304.

[Por3] T. Poreda, On generalized differential equations in Banach spaces, Dissertationes Mathematicae 310 (1991), 1-50.

[Re-Ma] F. Ren and J. Ma, Quasiconformal extension of biholomorphic mappings of several complex variables, J. Fudan Univ. Natur. Sci. 34 (1995), 545-556.

[Su] T.J. Suffridge, Starlike and convex maps in Banach spaces, Pacif. J. Math. 46 (1973), $575-589$.

H. Hamada

Faculty of Engineering

Kyushu Kyoritsu University

1-8 Jiyugaoka, Yahatanishi-ku

Kitakyushu 807-8585, Japan

Current address:

Faculty of Engineering

Kyushu Sangyo University

3-1 Matsukadai 2-Chome

Higashi-ku Fukuoka 813-8503, Japan

E-mail, H. Hamada: hamada@kyukyo-u.ac.jp

h.hamada@ip.kyusan-u.ac.jp

G. Kohr

Faculty of Mathematics

Babeş-Bolyai University

1 M. Kogălniceanu Str.

Cluj-Napoca, Romania

E-mail, G.Kohr: gkohr@math.ubbcluj.ro

Received: 08.01.2003 